\title{
nor-Mevaldic acid surrogates as selective antifungal agent leads against Botrytis cinerea. Enantioselective preparation of 4-hydroxy-6-(1-phenylethoxy)tetrahydro-2H-pyran-2-one
}

\author{
José Manuel Botubol-Ares a , María Jesús Durán-Peña ${ }^{a}$, Rosario Hernández-Galán ${ }^{\text {a }}$, Isidro G. Collado ${ }^{\text {a }}$, \\ Laurence M. Harwood ${ }^{\mathrm{b}}$, Antonio J. Macías-Sánchez ${ }^{\mathrm{a}, *}$ \\ a Departamento de Química Orgánica, Facultad de Ciencias, Campus Universitario Puerto Real, Universidad de Cádiz, Puerto Real, Cádiz 11510, Spain \\ ${ }^{\mathrm{b}}$ Department of Chemistry, University of Reading, Whiteknights, Reading RG6 6AD, United Kingdom
}

\section{A R T I C L E I N F O}

Article history:

Received 26 February 2015

Revised 13 April 2015

Accepted 15 April 2015

Available online 23 April 2015

\section{Keywords:}

Antifungal agents

Desymmetrisation

Lactones

nor-Methyl mevaldate

Statins

\begin{abstract}
A B S T R A C T
Solvent-free desymmetrisation of meso-dialdehyde $\mathbf{1}$ with chiral 1-phenylethan-1-ol, led to preparation of 4-silyloxy-6-alkyloxytetrahydro-2H-pyran-2-one (+)-3a with a 96:4 dr Deprotected lactone (+)-19a and the related racemic lactones $\mathbf{1 6 a - 1 8 a}$ present a lactone moiety resembling the natural substrate of HMG-CoA reductase and their antifungal properties have been evaluated against the phytopathogenic fungi Botrytis cinerea and Colletotrichum gloeosporioides. These compounds were selectively active against $B$. cinerea, while inactive against $C$. gloeosporioides.
\end{abstract}

(c) 2015 Elsevier Ltd. All rights reserved.

\section{Introduction}

Botrytis cinerea is a fungal pathogen which affects a wide range of cultivars. ${ }^{1}$ Variation in the attack modes, diversity of host and its survival ability, are on the basis of the difficulties found on the control of this phytopathogen. ${ }^{2}$ Use of chemical control agents against $B$. cinerea is hampered by the development of resistance ${ }^{3}$ to some active principles, such as benzimidazoles. ${ }^{4,5}$ On the other hand, there are public concerns on the safe use of chemical control agents and their impact in the environment, which involves tighter regulations on the use of new and established pesticides. ${ }^{6,7}$

Response to these problems involves a careful, coordinated use of chemical control agents, like application of mixed chemical fungicides spray progammes. ${ }^{8}$ Furthermore, there is considerable interest in the development of compounds which are compatible with integrated pest management practices; for instance these compounds should be compatible with the use of plant defense system activators such as acibenzolar, an analogue of salicylic acid, ${ }^{9}$ and biological control. ${ }^{6}$

Interaction between $B$. cinerea and the host is considered to involve the production of necrosis inducing factors that include proteins, reactive oxygen species, cell wall degrading enzymes,

\footnotetext{
* Corresponding author. Tel.: +34 956 012704; fax: +34 956016193.

E-mail address: antoniojose.macias@uca.es (A.J. Macías-Sánchez).
}

peptidases, phytohormones and phytotoxic metabolites like botrydial and botcinic acid, ${ }^{10-12}$ which are considered to be virulence factors (strain dependent).

Our research group has explored for some years a strategy of selective control of Botrytis cinerea through interference with virulence factors such as fungal toxins. ${ }^{13}$ Studies on the fungal toxins from $B$. cinerea have shown their diverse biosynthetic origins. Botrydial and related compounds originated from mevalonate pathway through FPP and further cyclization. ${ }^{14-17}$ Botcinins ${ }^{18}$ originated through poliketide biosynthetic pathway. ${ }^{19,20}$ Gene silencing studies have shown that blockade of one toxin metabolic pathway does not completely eliminate the pathogenicity of the fungus, due to the metabolic switch to another fungal toxin pathway. ${ }^{21,22}$

At the same time, genomic studies have been unraveling the complex metabolic potential of this versatile phytopathogen, showing that the genome of $B$. cinerea comprises 45 genes encoding secondary metabolites key enzymes including 22 polyketide synthases (PKSs), 8 non-ribosomal peptide synthetases (NRPSs), 5 PKS/NRPS, six sesquiterpene cyclases (STCs), three diterpene cyclases (DTCs) and one dimethylallyl tryptophan synthase (DMATS). ${ }^{23}$ As shown in the botrydial/botcinic acid switch, role and expression conditions of the cryptic metabolites encoded by the above mentioned genes may shed some light on the infection mechanisms of the fungus. 
Much of our previous work has been focused on the development of metabolic analogues to botrydial biosynthetic intermediates as a way to control the fungus. ${ }^{13}$ This has allowed the preparation of sesquiterpene lead compounds as antifungal agents. ${ }^{24}$ As shown by the genomic analysis, other terpenoids may be involved in the infective stages of the development of the fungus. Therefore, further work on the control of the fungus through inhibition of toxin production requires the preparation of compounds developed as selective inhibitors of the mevalonate pathway at an earlier stage in the biosynthetic pathway of sesquiterpenic toxins like botridial, so further studies could be developed on non mutant specimens. Furthermore, inhibition of mevalonate pathway should affect the production of constitutive steroids like ergosterol. Therefore, such compounds would have potential as antifungal agents, either on their own or in combination with other compounds.

Statins are a family of compounds that inhibit the formation of mevalonate through of the inhibition of the 3-hydroxy-3-methylglutaryl-CoA (HMG-CoA) reductase enzyme. Endo's original hypothesis ${ }^{25}$ for the development of HMG-CoA reductase selective inhibitors considered that those inhibitors found from microbial sources could be also applied to the inhibition of human HMGCoA reductase. This led to the discovery of compactin and development of the statins, either from natural or synthetic sources, ${ }^{26}$ which find widespread use in treatment of high triglyceride levels ${ }^{27}$ and hyperlipidemia in humans. ${ }^{28}$ As shown in recent findings of the inhibitory action of statins such as simvastatin and atorvastatin on the growth of Aspergillus fumigatus and Candida fungi, ${ }^{29}$ where fungal growth is recovered once ergosterol is provided exogenously, this reasoning may work in both ways. Moreover, quite likely, not all fungal HMG-CoA reductases are expected to have the same affinity for the above mentioned statins, and therefore they will not affect either fungal growth or sesquiterpenoid production. An example is the lack of significative effect on fungal growth of lovastatin when evaluated against B. cinerea. ${ }^{30}$

Therefore, the development of inhibitors of mevalonate pathway with potential antifungal activity against $B$. cinerea requires the preparation of novel lead compounds, related to known statins, which could be further developed into agents for the control of the fungus.

Statins show a common structural pattern, wherein a hydroxylactone moiety, reminiscent of mevalonic acid, is connected to a carbocyclic or heterocyclic fragment through an X-Y chain, where

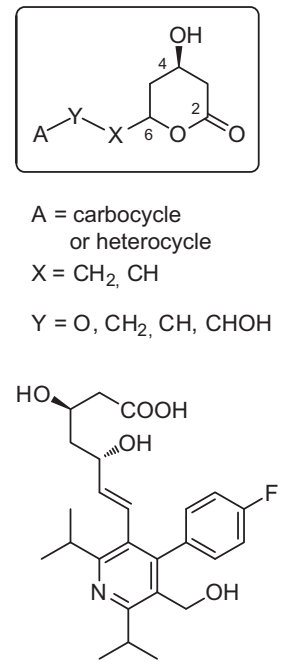

Cerivastatin

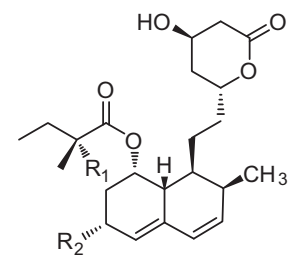

$\mathrm{R}_{1}=\mathrm{R}_{2}=\mathrm{H} ; \quad$ Compactin

$\mathrm{R}_{1}=\mathrm{R}_{2}=\mathrm{CH}_{3} ;$ Simvastatin

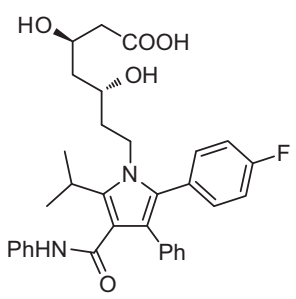

Atorvastatin
Figure 1. General structural pattern of representative statins. more frequently $\mathrm{X}$ and $\mathrm{Y}$ can be $\mathrm{CH}_{2}$, like in compactin, simvastatin or atorvastatin, or can be methyne groups like in cerivastatin. Furthermore, there are examples of active compounds where $\mathrm{X}$ is $\mathrm{CH}_{2}$ and $\mathrm{Y}$ is oxygen (Fig. 1). ${ }^{31}$ In addition, the stereochemistry of the stereogenic centers involved is defined and must be taken into account. To our knowledge there are no precedents for the preparation and evaluation of compounds with $\mathrm{X}=\mathrm{O}$.

As part of our strategy for the design of antifungal agents against Botrytis and Colletotrichum species, ${ }^{13}$ we report the preparation of 6-alkyloxy-4-hydroxytetrahydro-2H-pyran-2-ones (Fig. 1, $\mathrm{X}=\mathrm{O}, \mathrm{Y}=$ alkyl, $\mathrm{A}=$ aryl) which present a lactone moiety resembling the natural substrate of HMG-CoA reductase and the evaluation of the antifungal activities of selected substrates, either enantiomerically pure or racemic. Enantiomerically pure compounds are obtained via the desymmetrisation ${ }^{32}$ reaction of a suitable meso-dialdehyde ${ }^{33}$ precursor (1) with a chiral alcohol. ${ }^{34}$

\section{Results and discussion}

\subsection{Optimization of desymmetrisation reaction}

Enantioselective preparation of methyl 3-(tert-butyldimethylsilyloxy)-5-oxopentanoates (methyl nor-mevaldate derivatives) has been previously described in earlier reports from our research group through desymmetrisation of the dialdehyde 1 with (+)(R)-1-phenylethan-1-ol (2) to afford chiral 6-arylalkyloxy-4-silyloxytetrahydro-2H-pyran-2-ones (+)-3a and (+)-3b in a 9:1 dr (Scheme 1). ${ }^{34}$ The absolute stereochemistries of lactones $(+)-\mathbf{3 a}$ and (+)-3b were established unequivocally by a combination of $\mathrm{X}$-ray crystallographic analyses of the structurally related lactones $(+)-5 \mathbf{a}$ and $(-)-\mathbf{5 c}$, NOE difference studies and chemical correlation of lactones (+)-3a and (+)-5a with (-)-(R) methyl 3-(tertbutyldimethylsilyloxy)-5-oxopentanoate $(\mathbf{6})$ and of lactones $(+)$ 3b and (-)-5c with (+)-(S) methyl 3-(tert-butyldimethylsilyloxy)5-oxopentanoate (7) (Scheme 2). ${ }^{34}\left({ }^{1} \mathrm{H}\right.$ NMR data for compounds $\mathbf{3 a}, \mathbf{3 b},{ }^{34} \mathbf{5 a}$ and $\mathbf{5 c}$ in Table $3 ;{ }^{13} \mathrm{C}$ NMR data for compounds $\mathbf{3 a}$, $\mathbf{3 b},{ }^{34} \mathbf{5 a}$, and $\mathbf{5 c}$ in Table S3 of the SI).

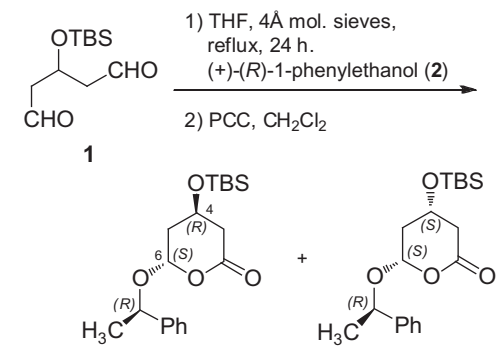

$(+)-3 \mathbf{a}(9 \%) \quad(+)-3 \mathbf{b}(1 \%)$

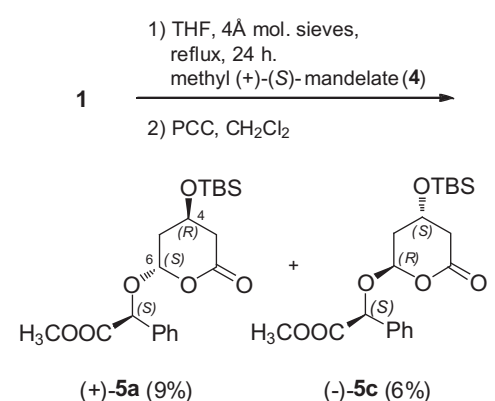

Scheme 1. Desymmetrisation of dialdehyde 1 by (R)-1-phenylethanol (2) and methyl (S)-mandelate (4). 
The configuration observed for the compounds $\mathbf{3 a}$ and $\mathbf{5 a}$ involves an axial distribution for the alkyloxy substituent on C-6 which would stabilize the configurations of the hemiacetal precursor with the axial exocyclic $\mathrm{C}-\mathrm{O}$ bond by the anomeric effect instead of the equatorial disposition which is present in the lactones $\mathbf{3 b}$ and $\mathbf{5 c}$ (Fig. 2). These conformations are consistent with those observed in many related substituted $\delta$-lactones. ${ }^{35}$

Applicability of these lactones required improvements in the number of steps needed for the preparation of the meso-precursor and in the diastereoselectivity of the desymmetrisation reaction. Dialdehyde 1, previously prepared in a 6-step synthetic sequence, ${ }^{34}$ was improved using an abridged synthetic sequence (Scheme 3) in 60\% overall yield, starting from hepta-1,6-dien-4ol, which was subjected to protection with $\mathrm{TBSCl}$, subsequent reductive ozonolysis and dehydration of the hydrate $\mathbf{9}{ }^{36}$

Efforts were then made to improve the yields and/or increase the selectivity of the desymmetrisation reaction using enantiomerically pure 1-phenylethanol, evaluating the influence of several catalysts and reagents. A slight increase in the overall yield was observed when a catalytic amount of $p$-toluenesulfonic acid, tin (II) triflate or 1 equiv of $N, N^{\prime}$-diisopropylethylamine (DIPEA) were employed (Table 1, entries 3, 4 and 8), but unfortunately at the cost of a decrease in the diastereoselectivity in the production of compounds $3 \mathbf{a}$ and $\mathbf{3 b}$. The addition of catalytic amounts of modified cinchona alkaloids such (DHQD) $)_{2} \mathrm{AQN}$ and (DHQD) ${ }_{2} \mathrm{PHAL}^{37}$ (Table 1, entries 5 and 6) or $\mathrm{Cr}$ (III) $\mathrm{Salen}^{38}$ complexes (Table 1, entry 7) neither increased the yields nor improved the selectivity of the reaction.

Given the tendency of dialdehyde $\mathbf{1}$ to transform into its hydrated form, and in order to avoid the use of dry THF, we decided to evaluate the reaction under solvent-free conditions. ${ }^{39}$ Gratifyingly, treatment of the freshly reconstituted dialdehyde $\mathbf{1}$ with 2.9 equiv of $(R)$-1-phenylethanol and $4 \AA$ molecular sieves at room temperature under solvent-free conditions yielded lactone 3a in a 96:4 dr (92\% de) (Table 2, entry 4$),{ }^{40}$ an improvement on previously described conditions. ${ }^{34}$

\subsection{Preparation of 6-arylalkyloxy-4-hydroxytetrahydro-2H- pyran-2-ones for antifungal testing}

A selection of simple achiral arylethanols, related to 1-phenylethan-1-ol, with modifications in the aromatic ring, was chosen in order to prepare a range of 6-arylalkyloxy-4-hydroxytetrahydro-2H-pyran-2-one analogs of $\mathbf{3 a}$, as compounds which present a lactone moiety resembling the natural substrate of HMG-CoA reductase, in order to evaluate their antifungal properties. Thereby, dialdehyde $\mathbf{1}$ was treated, as described above, with alcohols 10-12 in the presence of $4 \AA$ molecular sieves at room temperature, except in the case of alcohol $\mathbf{1 1}$ where the reaction was heated to $40{ }^{\circ} \mathrm{C}$. The crude lactols thus obtained were subsequently oxidized with PCC to afford the lactones ( \pm )-13a-15a (Scheme 4). ${ }^{1} \mathrm{H}$ NMR spectroscopic data and coupling constants for $\mathrm{H}-3$ and $\mathrm{H}-5$ signals in these tetrahydro- $2 \mathrm{H}$-pyran-2-ones were comparable to those for the compounds $\mathbf{3 a}$ and $\mathbf{5 a}$ (Table 3), which allowed us

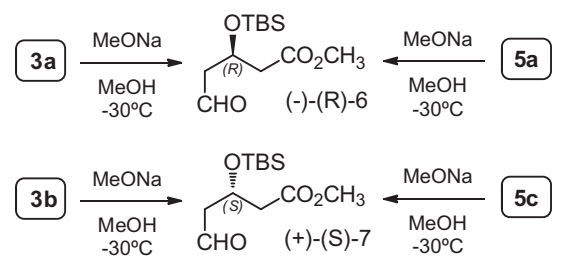

Scheme 2. Chemical correlation of lactones (+)-3a and (+)-5a with compound (-)$(R)-6$ and of lactones $(+)-3 \mathbf{b}$ and $(-)-5 \mathbf{c}$ with $(+)-(S)-7$.
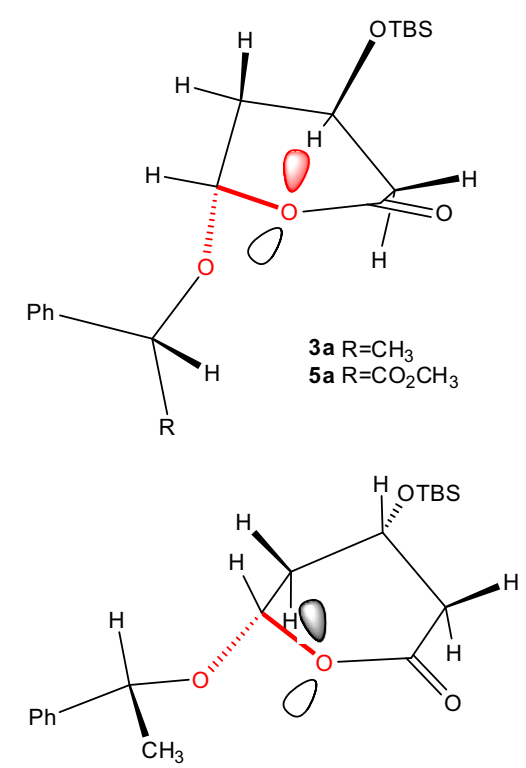

$3 \mathbf{b}$

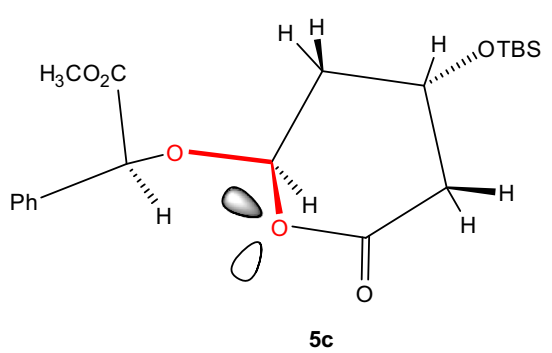

Figure 2. Most stable conformation for lactones $\mathbf{3 a}, \mathbf{3 b}, \mathbf{5 a}$ and $\mathbf{5 c}$.

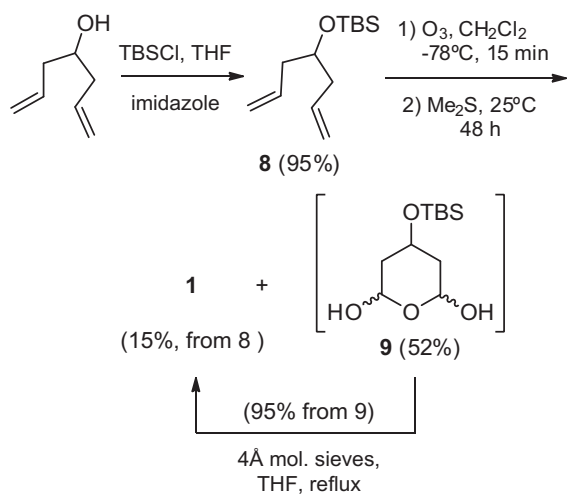

Scheme 3. Synthesis of the dialdehyde $\mathbf{1}$.

to assign the structures and relative stereochemistries for these compounds as $( \pm)-(3 R(S), 5 S(R))-6$-(arylalkyloxy)-4-(tert-butyldimethylsilyloxy)tetrahydro-2H-pyran-2-ones $(( \pm)-13 a-15 a) .\left({ }^{1} \mathrm{H}\right.$ NMR data for compounds 13a-15a in Table $3 ;{ }^{13} \mathrm{C}$ NMR data for compounds 13a-15a in Table S3 of the SI). Removal of the TBS group through treatment of compounds 13a-15a and 5a with the TBAF/ACOH ${ }^{41}$ system afforded the corresponding 6-arylalkyloxy-4hydroxytetrahydro- $2 H$-pyran-2-ones ( \pm -16a-18a and (+)-19a required for the essays, in moderate to good yields (Scheme 4). Treatment of compound $\mathbf{3 b}$ with the TBAF/AcOH ${ }^{41}$ system afforded (4S,6S)-4-hydroxy-6-((R)-1-phenylethoxy)tetrahydro-2H-pyran-2one $(\mathbf{1 9 b})$ in a $72 \%$ yield. 
Table 1

Desymmetrisation of dialdehyde 1 with either (R)-1-phenylethanol (2) or methyl $(S)$ mandelate (4) using catalysts/reagents ${ }^{\mathrm{a}}$

\begin{tabular}{lllll}
\hline Entry & Alcohol & Reagent & Products (\%) & $\mathrm{dr}^{\mathrm{b}}$ \\
\hline 1 & $\mathbf{2}$ & - & $\mathbf{3 a}(9), \mathbf{3 b}(1)$ & $90: 10^{34}$ \\
2 & $\mathbf{4}$ & - & $\mathbf{5 a}(9), \mathbf{5 c}(6)$ & $60: 40^{34}$ \\
3 & $\mathbf{2}$ & p-TsOH $(10 \mathrm{~mol} \%)$ & $\mathbf{3 a}(12), \mathbf{3 b}(3)$ & $80: 20$ \\
4 & $\mathbf{2}$ & Sn $(\mathrm{OTf})_{2}(10 \mathrm{~mol})$ & $\mathbf{3 a}(15), \mathbf{3 b}(3)$ & $83: 17$ \\
5 & $\mathbf{2}$ & $(\text { DHQD })_{2}$ PHAL $(10 \% \mathrm{~mol})^{\mathrm{c}}$ & $\mathbf{3 a}(9), \mathbf{3 b}(1)$ & $90: 10$ \\
6 & $\mathbf{2}$ & $(\text { DHQD })_{2}$ AQN $(10 \mathrm{~mol} \%)^{\mathrm{d}}$ & $\mathbf{3 a}(9), \mathbf{3 b}(1)$ & $90: 10$ \\
7 & $\mathbf{2}$ & (S,S) Cr$(\mathrm{III})$ Salen $\left(1\right.$ equiv $^{\mathrm{e}}$ & $\mathbf{3 a}(8), \mathbf{3 b}(2)$ & $80: 20$ \\
8 & $\mathbf{2}$ & DIPEA $(1 \text { equiv })^{\mathrm{f}}$ & $\mathbf{3 a}(17), \mathbf{3 b}(8)$ & $68: 32$ \\
\hline
\end{tabular}

a Yields obtained after chromatographic purification.

b Diasteroisomer ratios: ratio normalized to $100 \%$ between both diasteroisomers.

c $(\text { DHQD })_{2}$ PHAL = hydroquinidine 1,4-phthalazinediyl diether.

d $(\mathrm{DHQD})_{2} \mathrm{AQN}=$ hydroquinidine (anthraquinone-1,4-diyl) diether

e $(S, S) \mathrm{Cr}(\mathrm{III})$ Salen = (+)-(S,S)-N,N'-bis-(3,5-di-tert-butalicylidene)-(1,2-cyclohexanediamine) chromium (III) chloride.

${ }^{f} \mathrm{~N}, N^{\prime}$-Diisopropylethylamine.

Given that the structures of compounds $(+)-\mathbf{1 9 a}$ and $(+)-\mathbf{1 9 b}$ could be established, respectively, as (4R,6S)-4-hydroxy-6-( $(R)-1$ phenylethoxy)tetrahydro-2H-pyran-2-one and (4S,6S)-4-hydroxy6-((R)-1-phenylethoxy)tetrahydro-2H-pyran-2-one by chemical correlation, the structures and relative stereochemistries of compounds $( \pm)-\mathbf{1 6 a}-\mathbf{1 8 a}$ were assigned by comparison of spectroscopic data for deprotected lactone (+)-19a; this, in turn, confirmed the structure and relative stereochemistries assigned for parent compounds $( \pm)-13 a-15 a$. $\left({ }^{1} \mathrm{H}\right.$ NMR data for compounds 16a-18a, 19a and 19b in Table $4 ;{ }^{13} \mathrm{C}$ NMR data for compounds 16a-18a, 19a and 19b in Table S4 of the SI.)

\subsection{Antifungal activity}

The antifungal properties of compounds $( \pm)-\mathbf{1 6 a}-\mathbf{1 8 a}$ and $(+)-$ 19a, were determined against the growth of the phytopathogenic fungi Botrytis cinerea and Colletotrichum gloeosporioides at a concentration of $200 \mathrm{ppm}$ using the poisoned food technique ${ }^{42}$ (see fungal growth inhibition graphics, Figs. 3 and 4). The commercial fungicide Euparen ${ }^{\circledR}$ (dichlofluanid) was used as a standard for comparison in this test. Several levels of inhibition were observed (Figs. 3 and 4). Compounds ( \pm )-16a-18a and (+)-19a showed a substantial activity against $B$. cinerea (63-72\% fungal growth inhibition after 6 d, see Supporting information, Table S1). However, very little inhibition was observed against $C$. gloeosporioides. (Fig. 4, see also Supporting information, Table S2).

\section{Conclusions}

This selective inhibition of the growth of $B$. cinerea is of great interest as it opens the way to the design of selective fungicides against phytopathogenic fungi of the genus Botrytis and a potential integrated control of the fungus by combining selective fungicides and antagonistic fungi such as Trichoderma species, ${ }^{43}$ which

Table 2

Optimization of solvent-free desymmetrisation of dialdehyde $\mathbf{1}$ with $(R)$-1-phenylethanol (2)

\begin{tabular}{llllll}
\hline Entry & $\mathbf{2}$ (equiv) & $\mathbf{3 a}^{\mathrm{a}}(\%)$ & $\mathbf{3 b}^{\mathrm{a}}(\%)$ & $\mathrm{dr}^{\mathrm{b}}$ & $\mathrm{de}^{\mathrm{c}}(\%)$ \\
\hline 1 & 9.5 & 5 & 1.2 & $80: 20$ & 60 \\
2 & 5.0 & 7.5 & 1.4 & $84: 16$ & 68 \\
3 & 3.5 & 7.1 & 0.9 & $89: 11$ & 78 \\
4 & 2.9 & 12.3 & 0.6 & $96: 4$ & 92 \\
\hline
\end{tabular}

\footnotetext{
a Yields obtained after chromatographic purification.

b Diasteroisomer ratios: ratio normalized to $100 \%$ between both diasteroisomers.

c $\mathrm{de} \%=100(\mathbf{3} \mathbf{a} \%-\mathbf{3 b} \%) /(\mathbf{3} \mathbf{a} \%+\mathbf{3 b} \%)$
}

potentially would not be affected by the fungicides aimed at Botrytis. ${ }^{44,45}$ The mode of action of these compounds may be related with the inhibition of the biosynthesis of terpenes, such as the sesquiterpene phytotoxin botrydial, ${ }^{13}$ and this will be further explored. Interestingly, no terpenes have been reported from Colletotrichum spp., except for Colletotrichum nicotiniae. ${ }^{46}$

\section{Experimental section}

\subsection{General}

Unless otherwise noted, materials and reagents were obtained from commercial suppliers and were used without further purification. Dichloromethane was freshly distilled from $\mathrm{CaH}_{2}$ and tetrahydrofuran was dried over sodium and benzophenone and freshly distilled before use. Air- and moisture-sensitive reactions were performed under an argon atmosphere. Melting points were measured with a Reichert-Jung Kofler block and are uncorrected. Optical rotations were determined on a Perkin-Elmer 341 polarimeter. IR spectra were recorded on a Perkin-Elmer Spectrum BX FT-IR spectrophotometer. ${ }^{1} \mathrm{H}$ and ${ }^{13} \mathrm{C}$ NMR spectra were obtained on Varian Gemini 300, Varian INOVA 400 and Agilent $500 \mathrm{MHz}$ NMR spectrometers using tetramethylsilane as an internal reference. NMR assignments were made by a combination of 1D and 2D techniques and by comparison with assignments available in the literature for previously described compounds, where appropriate. Mass spectra were recorded on a Finnigan Voyager spectrometer at $70 \mathrm{eV}$. High resolution mass spectra were recorded on a Micromass Autospec spectrometer at $70 \mathrm{eV}$ or in a Waters Synapt G2 QTOF mass spectrometer in positive ion electrospray mode at $20 \mathrm{~V}$ cone voltage. HPLC was performed with a
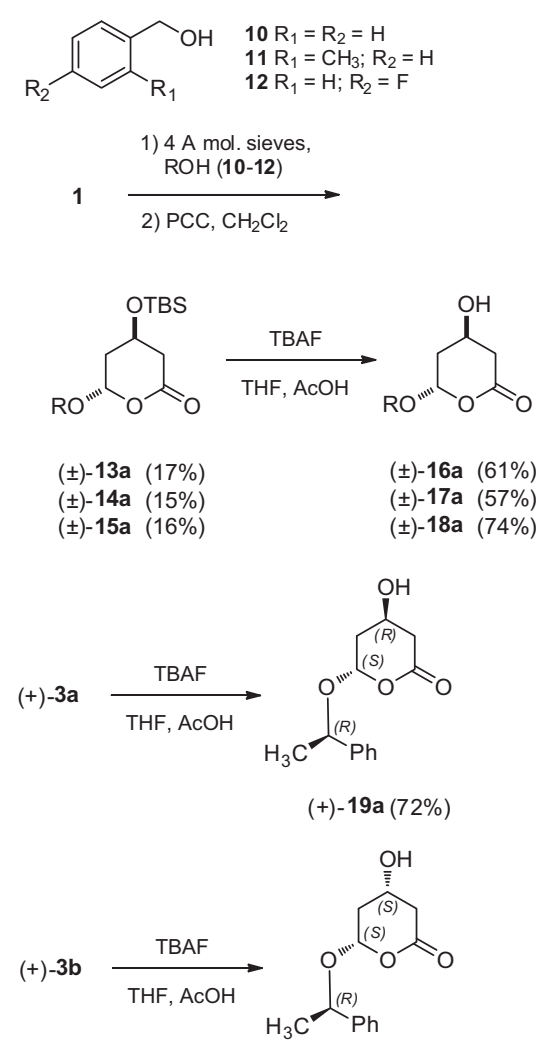

$(+)-19 b(72 \%)$

Scheme 4. Preparation of deprotected lactones and ( \pm )-16a-18a, $(+)-19 a$ and $(+)-$ 19b. 
Table 3

${ }^{1} \mathrm{H}$ NMR spectroscopic data for 3a, 5a, 3b, 5c, $( \pm)-\mathbf{1 3 a},( \pm)-\mathbf{1 4 a},( \pm)-\mathbf{1 5} \mathbf{a}$

\begin{tabular}{|c|c|c|c|c|c|c|c|}
\hline Position & $\begin{array}{l}3_{\mathbf{a}^{34}} \\
\delta_{\mathrm{H}}(\text { mult, } J \text { in } \mathrm{Hz})^{\mathrm{a}}\end{array}$ & $\begin{array}{l}\mathbf{5 a} \\
\delta_{\mathrm{H}}(\text { mult }, J \text { in } \mathrm{Hz})^{\mathrm{b}}\end{array}$ & $\begin{array}{l}\mathbf{3 b}^{34} \\
\delta_{\mathrm{H}}(\text { mult, } J \text { in } \mathrm{Hz})^{\mathrm{a}}\end{array}$ & $\begin{array}{l}\mathbf{5 c} \\
\delta_{\mathrm{H}}(\text { mult, } J \text { in } \mathrm{Hz})^{\mathrm{c}}\end{array}$ & $\begin{array}{l}( \pm)-\mathbf{1 3 a} \\
\delta_{\mathrm{H}}(\text { mult, } J \text { in } \mathrm{Hz})^{\mathrm{a}}\end{array}$ & $\begin{array}{l}( \pm)-\mathbf{1 4 a} \\
\delta_{\mathrm{H}}(\text { mult, } J \text { in } \mathrm{Hz})^{\mathrm{a}}\end{array}$ & $\begin{array}{l}( \pm)-\mathbf{1 5 a} \\
\delta_{\mathrm{H}}(\text { mult, } J \text { in } \mathrm{Hz})^{\mathrm{a}}\end{array}$ \\
\hline 3 & $\alpha: 2.47, \mathrm{dd},(17.2,5.6)$ & $\alpha: 2.50, \mathrm{dd},(17.2,5.6)$ & $\alpha: 2.51, \mathrm{dd},(16.8,9.2)$ & $\alpha: 2.50, \mathrm{dd},(17.3,4.8)$ & $\alpha: 2.52, \mathrm{dd},(17.4,5.6)$ & $\alpha: 2.51, \mathrm{dd},(17.4,5.6)$ & $\alpha: 2.51, \mathrm{dd},(17.2,5.4)$ \\
\hline & $\beta: 2.76, \mathrm{dd},(17.2,4.8)$ & $\beta: 2.85$, ddd, $(17.2,4.8,1.2)$ & $\beta: 2.69$ ddd, $(16.8,5.4,1.8)$ & $\beta: 2.71 \mathrm{dd},(17.3,4.8)$ & $\beta: 2.78 \mathrm{dd},(17.4,4.8)$ & $\beta: 2.78 \mathrm{dd},(17.4,4.8)$ & $\beta: 2.76 \mathrm{dd},(17.2,4.6)$ \\
\hline 4 & $4.33, \mathrm{~m}$ & 4.44, $\mathrm{m}$ & $3.98, \mathrm{tt}(9.2,5.4)$ & 4.38 , quint (4.8) & $4.36, \mathrm{~m}$ & $4.36, \mathrm{~m}$ & $4.35, \mathrm{~m}$ \\
\hline 5 & $\begin{array}{l}\alpha: 1.94, \text { ddd }(13.8, \\
5.6,3.6) \\
\beta: 1.85, \text { ddd }(13.8, \\
6.8,3.6)\end{array}$ & $\begin{array}{l}\alpha: 2.13, \operatorname{dddd}(14.0,4.8,3.8,1.2) \\
\beta: 1.95, \text { ddd }(14.0,6.8,3.8)\end{array}$ & $\begin{array}{l}\alpha 1.82, \operatorname{ddd}(14.0,9.2,7.7) \\
\beta: 2.22, \operatorname{dddd}(14.0,5.4,4.4,1.8)\end{array}$ & $\alpha, \beta: 2.17, \mathrm{t}(4.8)$ & $\alpha, \beta: 2.05-1.95$ & $\alpha, \beta: 2.06-1.95$ & $\alpha, \beta: 2.00, \mathrm{t}(4.4)$ \\
\hline 6 & $5.25, \mathrm{dd}(5.6,3.6)$ & $5.37, \mathrm{dd}(4.8,3.8)$ & $5.04, \mathrm{dd}(7.7,4.4)$ & $5.64, \mathrm{t}(4.8)$ & $5.50, \mathrm{dd}(5.2,4.0)$ & $5.51, \mathrm{dd}(5.2,4.0)$ & $5.48, \mathrm{t}(4.4)$ \\
\hline $\mathrm{Si}\left(\mathrm{CH}_{3}\right)\left(\mathrm{CH}_{3}\right)$ & $0.02, s$ & $0.04, \mathrm{~s}$ & $0.03, \mathrm{~s}$ & $0.09, \mathrm{~s}$ & $0.06, \mathrm{~s}$ & $0.06, \mathrm{~s}$ & $0.05, \mathrm{~s}$ \\
\hline $\mathrm{Si}\left(\mathrm{CH}_{3}\right)\left(\mathrm{CH}_{3}\right)$ & $-0.03, \mathrm{~s}$ & $0.00, \mathrm{~s}$ & $0.03, \mathrm{~s}$ & $0.08, \mathrm{~s}$ & $0.04, \mathrm{~s}$ & $0.05, \mathrm{~s}$ & $0.04, \mathrm{~s}$ \\
\hline $\mathrm{SiC}\left(\mathrm{CH}_{3}\right)_{3}$ & $0.76, \mathrm{~s}$ & $0.79, \mathrm{~s}$ & $0.86, \mathrm{~s}$ & $0.88, \mathrm{~s}$ & $0.84, \mathrm{~s}$ & $0.86, \mathrm{~s}$ & $0.84, \mathrm{~s}$ \\
\hline $2^{\prime}$ & $7.36-7.28$ & $7.44-7.36$ & $7.38-7.28$ & $7.40-7.35$ & $7.37-7.29$ & - & $7.32-7.26$ \\
\hline $3^{\prime}$ & $7.36-7.28$ & $7.44-7.36$ & $7.38-7.28$ & $7.40-7.35$ & $7.37-7.29$ & 7.19-7.17, m & $7.05-7.01$ \\
\hline $4^{\prime}$ & $7.36-7.28$ & 7.44-7.36 & $7.38-7.28$ & $7.40-7.35$ & $7.37-7.29$ & $7.24-7.20$ & - \\
\hline $5^{\prime}$ & $7.36-7.28$ & 7.44-7.36 & $7.38-7.28$ & $7.40-7.35$ & $7.37-7.29$ & 7.24-7.20 & $7.05-7.01$ \\
\hline $6^{\prime}$ & $7.36-7.28$ & $7.44-7.36$ & $7.38-7.28$ & $7.40-7.35$ & $7.37-7.29$ & $7.31-7.28, \mathrm{~m}$ & $7.32-7.26$ \\
\hline$o-\mathrm{CH}_{3} \mathrm{PhCH}_{2} \mathrm{O}$ & - & - & - & & - & $2.32, \mathrm{~s}$ & - \\
\hline $\mathrm{ArCH}(\mathrm{H}) \mathrm{O}$ & - & - & - & & $4.93, \mathrm{~d}(11.8)$ & $4.97, \mathrm{~d}(11.8)$ & $4.89, \mathrm{~d}(11.8)$ \\
\hline $\operatorname{ArCH}(H) \mathrm{O}$ & - & - & - & & $4.63, \mathrm{~d}(11.8)$ & $4.61, \mathrm{~d}(11.8)$ & $4.60, \mathrm{~d}(11.8)$ \\
\hline $\mathrm{CH}_{3} \mathrm{OCO}$ & - & $3.69, \mathrm{~s}$ & - & $3.72, \mathrm{~s}$ & - & - & - \\
\hline $\mathrm{ArCH}\left(\mathrm{CH}_{3}\right) \mathrm{O}$ & $1.46, \mathrm{~d}(6.6)$ & - & $1.47, \mathrm{~d}(6.6)$ & & - & - & - \\
\hline $\mathrm{ArCH}\left(\mathrm{R}_{1}\right) \mathrm{O}$ & $4.99, \mathrm{q}(6.6)$ & $5.32, \mathrm{~s}$ & $5.01, \mathrm{q}(6.6)$ & $5.48, \mathrm{~s}$ & - & - & - \\
\hline
\end{tabular}

a Acquired in $\mathrm{CDCl}_{3}(400 \mathrm{MHz})$.

Acquired in $\mathrm{CDCl}_{3}(300 \mathrm{MHz})$.

Acquired in $\mathrm{CDCl}_{3}(500 \mathrm{MH})$

Interchangeable signals. 
Table 4

${ }^{1} \mathrm{H}$ NMR spectroscopic data for $( \pm)-\mathbf{1 6 a}-\mathbf{1 8 a}, \mathbf{1 9 a}$ and $\mathbf{1 9 b}$

\begin{tabular}{|c|c|c|c|c|c|}
\hline Position & $\begin{array}{l}( \pm)-\mathbf{1 6 a} \\
\delta_{\mathrm{H}}(\text { mult, } J \text { in } \mathrm{Hz})^{\mathrm{a}}\end{array}$ & $\begin{array}{l}( \pm)-\mathbf{1 7 a} \\
\delta_{\mathrm{H}}(\text { mult, } J \text { in } \mathrm{Hz})^{\mathrm{a}}\end{array}$ & $\begin{array}{l}( \pm)-\mathbf{1 8 a} \\
\delta_{\mathrm{H}}(\text { mult, } J \text { in } \mathrm{Hz})^{\mathrm{a}}\end{array}$ & $\begin{array}{l}\text { 19a } \\
\delta_{\mathrm{H}}(\text { mult, } J \text { in } \mathrm{Hz})^{\mathrm{a}}\end{array}$ & $\begin{array}{l}\text { 19b } \\
\delta_{\mathrm{H}}(\text { mult, } J \text { in } \mathrm{Hz})^{\mathrm{a}}\end{array}$ \\
\hline 3 & $\begin{array}{l}\alpha: 2.54, \text { ddd, }(17.6,6.4,0.8) \\
\beta: 2.92, \text { ddd, }(17.6,5.2,1.2)\end{array}$ & $\begin{array}{l}\alpha: 2.51, \text { ddd, }(17.4,6.4,0.8) \\
\beta: 2.87, \text { ddd, }(17.4,5.2,0.8)\end{array}$ & $\begin{array}{l}\alpha: 2.55, \text { ddd, }(17.6,6.2,0.8) \\
\beta: 2.91, \text { ddd, }(17.6,5.2,0.8)\end{array}$ & $\begin{array}{l}\alpha: 2.51, \mathrm{ddd},(17.4,6.4,0.8) \\
\beta: 2.94, \mathrm{ddd},(17.4,5.6,1.2)\end{array}$ & $\alpha, \beta: 2.84$ \\
\hline 4 & $4.46, \mathrm{~m}$ & $4.40, \mathrm{~m}$ & $4.45, \mathrm{~m}$ & $4.48, \mathrm{~m}$ & $\begin{array}{l}4.23 \text {, dtt }(8.8,5.2 \text {, } \\
4.0)\end{array}$ \\
\hline 5 & $\begin{array}{l}\alpha: 2.02, \operatorname{dddd}(13.8,7.6,4.0 \text {, } \\
0.8) \\
\beta: 2.18, \operatorname{ddt}(13.8,4.0,1.2)\end{array}$ & $\begin{array}{l}\alpha: 1.98, \text { dddd }(14.0,7.6,4.0 \text {, } \\
0.8) \\
\beta: 2.12 \text {, ddt }(14.0,4.0,0.8)\end{array}$ & $\begin{array}{l}\alpha: 2.03, \operatorname{dddd}(13.6,7.6,4.2 \text {, } \\
0.8) \\
\beta: 2.16, \operatorname{ddt}(13.6,4.2,1.2)\end{array}$ & $\begin{array}{l}\alpha: 1.89, \operatorname{dddd}(14.0,8.0,4.0 \text {, } \\
0.8) \\
\beta: 2.11, \operatorname{ddt}(14.0,4.0,1.2)\end{array}$ & $\alpha, \beta: 2.09$ \\
\hline 6 & $5.53, \mathrm{t}(4.0)$ & $5.50, \mathrm{t}(4.0)$ & $5.52, \mathrm{t}(4.2)$ & $5.26, \mathrm{t}(4.0)$ & $5.25, \mathrm{t}(4.0)$ \\
\hline $2^{\prime}$ & $7.37-7.30$ & - & $7.31-7.27$ & $7.37-7.27$ & $7.37-7.35$ \\
\hline $3^{\prime}$ & $7.37-7.30$ & $7.16-7.14$ & $7.06-7.01$ & $7.37-7.27$ & $7.33-7.30$ \\
\hline $4^{\prime}$ & $7.37-7.30$ & $7.22-7.17$ & - & $7.37-7.27$ & $7.33-7.30$ \\
\hline $5^{\prime}$ & $7.37-7.30$ & $7.22-7.17$ & $7.06-7.01$ & $7.37-7.27$ & $7.33-7.30$ \\
\hline $6^{\prime}$ & $7.37-7.30$ & $7.26-7.24$ & $7.31-7.27$ & $7.37-7.27$ & $7.37-7.35$ \\
\hline $\mathrm{o}-\mathrm{CH}_{3} \mathrm{PhCH}_{2} \mathrm{O}$ & - & $2.28, \mathrm{~s}$ & - & - & - \\
\hline $\mathrm{ArCH}(\mathrm{H}) \mathrm{O}$ & $4.90, \mathrm{~d}(11.8)$ & $4.91, \mathrm{~d}(11.6)$ & $4.85, \mathrm{~d}(11.6)$ & - & - \\
\hline $\operatorname{ArCH}(H) \mathrm{O}$ & $4.62, \mathrm{~d}(11.8)$ & $4.55, \mathrm{~d}(11.6)$ & $4.57, \mathrm{~d}(11.6)$ & - & - \\
\hline $\mathrm{ArCH}\left(\mathrm{CH}_{3}\right) \mathrm{O}$ & - & - & - & $1.43, \mathrm{~d}(6.4)$ & $1.50, \mathrm{~d}(6.6)$ \\
\hline $\mathrm{ArCH}\left(\mathrm{CH}_{3}\right) \mathrm{O}$ & - & - & - & $4.96, \mathrm{q}(6.4)$ & $5.04, \mathrm{q}(6.6)$ \\
\hline $\mathrm{OH}$ & - & - & - & - & $3.25, \mathrm{~d}(8.8)$ \\
\hline
\end{tabular}

a Acquired in $\mathrm{CDCl}_{3}(400 \mathrm{MHz})$.

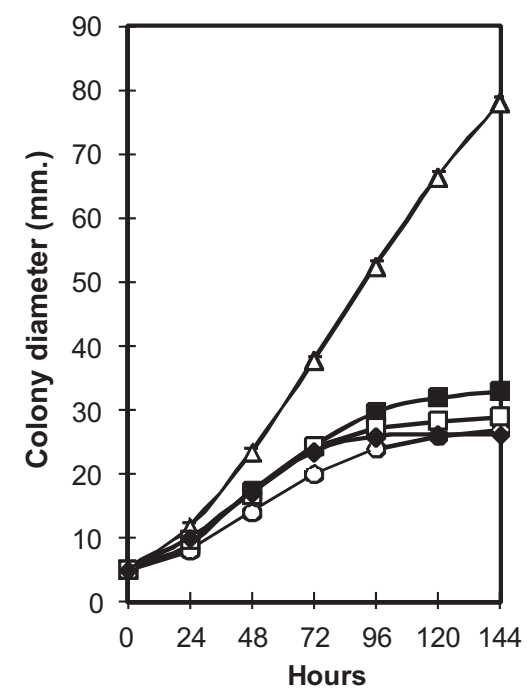

$\rightarrow-$ Control $\rightarrow-16 a \quad \rightarrow-17 a$
$-\square-18 a \quad \longrightarrow 19 a$

Figure 3. Comparison of fungal growth inhibition (B. cinerea) among compounds 16a-19a (200 ppm dose; 16a $\left(0.9010^{-3} \mathrm{M}\right), 17 \mathbf{a}, 19 a\left(0.8510^{-3} \mathrm{M}\right)$ and 18a $\left(0.8310^{-3} \mathrm{M}\right)$

Hitachi/Merck L-6270 apparatus equipped with a UV-VIS detector (L 4250) and a differential refractometer detector (RI-71). TLC was performed on Merck Kiesegel 60 F254, $0.2 \mathrm{~mm}$ thick. Silica gel (Merck) was used for column chromatography. Purification by HPLC was performed using a Si gel column (LiChrospher Si 60, $10 \mu \mathrm{m}, 1 \mathrm{~cm}$ wide, $25 \mathrm{~cm}$ long).

\subsection{Microorganism and antifungal assays}

The culture of Botrytis cinerea strain UCA 992 employed in this work was isolated from Domecq vineyard grapes, Jerez de la Frontera, Cádiz, Spain. This culture of B. cinerea has been deposited at the Mycological Herbarium Collection (UCA), Facultad de Ciencias, Universidad de Cádiz. The culture of Colletotrichum gloeosporioides utilized, C. gloeosporioides CECT 20122, was

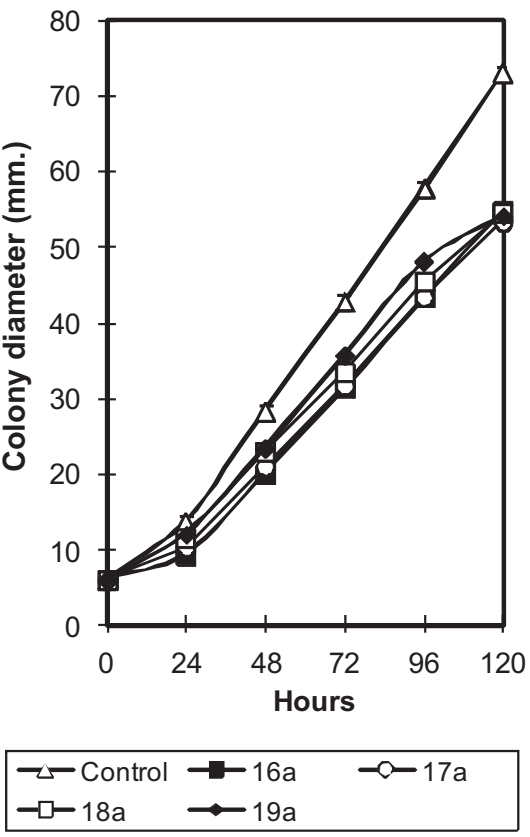

Figure 4. Comparison of fungal growth inhibition (C. gloeosporioides) among 16a19a (200 ppm dose; 16a $\left(0.9010^{-3} \mathrm{M}\right), \mathbf{1 7 a}, \mathbf{1 9 a}\left(0.8510^{-3} \mathrm{M}\right)$ and 18a $\left(0.8310^{-3} \mathrm{M}\right)$.

obtained from the Colección Española de Cultivos Tipo Collection Depository. The growth medium was poured into $9 \mathrm{~cm}$ diameter sterile plastic petri dishes, and a $5 \mathrm{~mm}$ diameter mycelial disk of $B$. cinerea or $C$. gloeosporioides cut from an actively growing culture was placed in the center of the plate. Antifungal bioassays were performed by measuring radial growth on agar medium for $B$. cinerea and potato-dextrose-agar (PDA) for $C$. gloeosporioides in the presence of test compounds. Test compounds were dissolved in EtOH to a final compound concentration in the culture medium of $200 \mathrm{mg} \mathrm{L}^{-1}$. The final EtOH concentration was identical in both the control and treated cultures. Radial growth was measured for five days for $C$. gloeosporioides and for 6 days for B. cinerea; experiments were carried out at $25^{\circ} \mathrm{C}$, in triplicate. The percentage 
inhibitions of mycelial growth over controls were calculated using the following formula. ${ }^{47}$

$$
\begin{aligned}
& I(\%)=\frac{(C-T)}{\left(C-T_{0}\right)} \times 100 \\
& I=\text { Inhibition rate } \\
& C=\text { Diameter of control colony } \\
& T=\text { Diameter of treated colony } \\
& T_{0}=\text { Initial diameter of treated colony }
\end{aligned}
$$

\subsection{Procedures}

\subsubsection{Preparation of 4-(tert-butyldimethylsilyloxy)hepta-1,6- diene (8)}

A solution of tert-butyldimethylsilane chloride (TBSCl) $(4.2 \mathrm{~g}$, $26.8 \mathrm{mmol})$ in dry THF $(7 \mathrm{~mL})$ was added to a stirred solution of imidazole $(8.6 \mathrm{~g}, 142.9 \mathrm{mmol})$ and hepta-1,6-dien-4-ol $(2.0 \mathrm{~g}$, $17.86 \mathrm{mmol})$ in dry THF $(25 \mathrm{~mL})$ at $0{ }^{\circ} \mathrm{C}$ under an argon atmosphere. The mixture was allowed to warm to room temperature and when TLC analysis indicated completion of reaction $(16 \mathrm{~h})$ diethyl ether was added $(60 \mathrm{~mL})$. The organic layer was washed three times with brine $(40 \mathrm{~mL})$, dried over anhydrous sodium sulfate and filtered. Evaporation of the solvent gave a residue that was purified by silica gel column chromatography (petroleum ether/EtOAc, 90:10), to yield compound 8 (3.8 g, 95\%). Spectroscopic data for compound $\mathbf{9}$ were identical to those described in the literature. ${ }^{48}$

\subsubsection{Preparation of 3-(tert-butyldimethylsilyloxy)pentanedial} (1)

Ozone was bubbled through a stirred solution of 4-(tertbutyldimethylsilyloxy)hepta-1,6-diene (8) (3.2 g, $14.37 \mathrm{mmol})$ in $\mathrm{CH}_{2} \mathrm{Cl}_{2}(30 \mathrm{~mL})$ under an argon atmosphere at $-78^{\circ} \mathrm{C}$ until a permanent blue coloration was obtained ( $25 \mathrm{~min}$ ). Oxygen was subsequently bubbled through the solution ( $5 \mathrm{~min}$ ) and then dimethyl sulfide was slowly added $(18 \mathrm{~mL})$, which led to disappearance of the blue coloration, the reaction was allowed to warm to room temperature and stirred for further $48 \mathrm{~h}$. The organic layer was washed three times with brine $(40 \mathrm{~mL})$, dried over anhydrous sodium sulfate and filtered. Evaporation of the solvent under reduced pressure gave a residue $(3.3 \mathrm{~g})$ which was refluxed in dry THF $(150 \mathrm{~mL})$ in the presence of $4 \AA$ molecular sieves $(11.3 \mathrm{~g})$ for $16 \mathrm{~h}$. Then, the reaction was allowed to cool to room temperature, filtered through a pad of Celite ${ }^{\circledR}$ and the solvent evaporated under reduced pressure to yield 3-(tert-butyldimethylsilyloxy)pentanedial (1) (2115 mg, 64\%) as a colorless oil, which was utilized immediately without chromatographic purification. Spectroscopic data for compound $\mathbf{1}$ were identical to those described in the literature. ${ }^{36 \mathrm{~b}}$

\subsubsection{General procedure for the preparation of 6-alkyloxy-4- (tert-butyldimethylsilyloxy)tetrahydro-2H-pyran-2-ones} (3a and b, 5a and 5c): Desymmetrisation of 3-(tert-butyldimethylsilyloxy)pentanedial (1) under reflux followed by THF/PCC oxidation

Either $(R)$-1-phenylethanol (2.7 mmol) or (S)-methyl mandelate $(2.7 \mathrm{mmol})$ were added to a suspension of the reagents detailed in Table 1, dialdehyde $1(2.5 \mathrm{mmol})$ and $4 \AA$ powdered molecular sieves $(2.5 \mathrm{~g})$ in dry THF $(10 \mathrm{~mL})$. The slurry was refluxed for $24 \mathrm{~h}$, filtered through Celite ${ }^{\circledR}$ and the THF was removed under reduced pressure to yield an oil, which was dissolved in DCM $(12 \mathrm{~mL})$ and then added dropwise to a suspension of PCC (3.2 mmol) and powdered $4 \AA$ molecular sieves $(1.4 \mathrm{~g})$ in DCM $(15 \mathrm{~mL})$ at room temperature. The reaction was stirred vigorously at room temperature for $18 \mathrm{~h}$, diethyl ether was then added and the mixture was stirred for a further $1 \mathrm{~h}$. The suspension was filtered through a pad of silica gel, and washed through with further ether. The ether was removed under reduced pressure to give the crude mixture of tetrahydro-2H-pyran-2-ones. Further purification by column chromatography (petroleum ether/ $\mathrm{Et}_{2} \mathrm{O}$ 90:10) yielded the corresponding tetrahydro- $2 H$-pyran-2-ones $\mathbf{3 a}$, 3b (from $(R)$ 1-phenylethanol) or 5a and $\mathbf{5 c}$ (from (S)-methyl mandelate) in the yields and ratios shown in Table 1 .

4.3.3.1. (4R,6S)-4-((tert-butyldimethylsilyl)oxy)-6-((R)-1-phenylethoxy)tetrahydro-2H-pyran-2-one (3a) ${ }^{34}$. Colorless oil; HPLC $t_{\mathrm{R}}=41 \mathrm{~min}$ (Petroleum ether: ethyl acetate 93:7; flow $=3.0 \mathrm{~mL} / \mathrm{min}$ ); $[\alpha]_{D}^{20}+157^{\circ}$ (c 1.0, $\mathrm{CHCl}_{3}$ ); IR (film) $v_{\max }$ 2927, 2857, 1752, 1560, 1229, $1024 \mathrm{~cm}^{-1} ;{ }^{1} \mathrm{H}$ NMR $\left(\mathrm{CDCl}_{3}\right.$, $400 \mathrm{MHz}$ ) (see Table 3); ${ }^{13} \mathrm{C} \mathrm{NMR}\left(\mathrm{CDCl}_{3}, 100 \mathrm{MHz}\right)$ (see Table S3 of the SI); HRESIMS(+): $\mathrm{m} / \mathrm{z} 373.1812[\mathrm{M}+\mathrm{Na}]^{+}$(calcd for $\mathrm{C}_{19} \mathrm{H}_{30} \mathrm{O}_{4} \mathrm{NaSi}, 373.1811$ ); $\mathrm{m} / z$ 229.1262 [M+H- $\left.\mathrm{C}_{8} \mathrm{H}_{9} \mathrm{OH}\right]^{+}$(calcd for $\mathrm{C}_{11} \mathrm{H}_{21} \mathrm{O}_{5} \mathrm{Si}, 229.1260$ ).

4.3.3.2. $\quad 4 S, 6 S)-4-(($ tert-butyldimethylsilyl)oxy)-6-((R)-1-phenylethoxy)tetrahydro-2H-pyran-2-one $(\mathbf{3 b})^{34}$. Colorless oil; HPLC $t_{\mathrm{R}}=33 \mathrm{~min}$ (Petroleum ether: ethyl acetate 93:7; flow $=3.0 \mathrm{~mL} / \mathrm{min}$ ); $[\alpha]_{\mathrm{D}}^{20}+144^{\circ}$ (c $0.4, \mathrm{CHCl}_{3}$ ); IR (film) $v_{\max }$ 2927, 2857, 1752, 1560, 1229, $1024 \mathrm{~cm}^{-1} ;{ }^{1} \mathrm{H}$ NMR $\left(\mathrm{CDCl}_{3}\right.$, $400 \mathrm{MHz}$ ) (see Table 3$) ;{ }^{13} \mathrm{C}$ NMR $\left(\mathrm{CDCl}_{3}, 100 \mathrm{MHz}\right.$ ) (see Table S3 of the SI); HRESIMS $(+)$ : $m / z$ 373.1811 $[\mathrm{M}+\mathrm{Na}]^{+}$(calcd for $\mathrm{C}_{19} \mathrm{H}_{30} \mathrm{O}_{4} \mathrm{NaSi}, 373.1811$ ); $\mathrm{m} / \mathrm{z} 229.1261\left[\mathrm{M}+\mathrm{H}-\mathrm{C}_{8} \mathrm{H}_{9} \mathrm{OH}\right]^{+}$(calcd for $\mathrm{C}_{11} \mathrm{H}_{21} \mathrm{O}_{5} \mathrm{Si}, 229.1260$ ).

4.3.3.3. (S)-methyl 2-(((2S,4R)-4-((tert-butyldimethylsilyl)oxy)6-oxotetrahydro-2H-pyran-2-yl)oxy)-2-phenylacetate (5a). Yellow solid, mp. $99-101^{\circ} \mathrm{C}$; HPLC $t_{\mathrm{R}}=24$ min (Petroleum ether: ethyl acetate 88:12; flow $=3.5 \mathrm{~mL} / \mathrm{min}) ; \quad[\alpha]_{\mathrm{D}}^{20}+153^{\circ}$ (c 0.9 , $\mathrm{CHCl}_{3}$ ); IR (film) $v_{\max } 2954,2858,1757,1559,1211,1105 \mathrm{~cm}^{-1}$; ${ }^{1} \mathrm{H}$ NMR $\left(\mathrm{CDCl}_{3}, 300 \mathrm{MHz}\right)$ (see Table 3$) ;{ }^{13} \mathrm{C}$ NMR $\left(\mathrm{CDCl}_{3}\right.$, $75 \mathrm{MHz}$ ) (see Table S3 of the SI); HRESIMS(+): $m / z 417.1709$ $[\mathrm{M}+\mathrm{Na}]^{+}$(calcd for $\mathrm{C}_{20} \mathrm{H}_{30} \mathrm{O}_{6} \mathrm{NaSi}, 417.1705$ ); $\mathrm{m} / z 263.0917$ [M+H$\left.\mathrm{C}_{6} \mathrm{H}_{15} \mathrm{SiOH}\right]^{+}$(calcd for $\mathrm{C}_{14} \mathrm{H}_{15} \mathrm{O}_{5}, 263.0919$ ).

4.3.3.4. (S)-methyl $2-(((2 R, 4 S)-4-((t e r t-b u t y l d i m e t h y l s i l y l) o x y)-$ 6-oxotetrahydro-2H-pyran-2-yl)oxy)-2-phenylacetate (5c). Yellow solid, m.p. $82-84^{\circ} \mathrm{C}$; HPLC $t_{\mathrm{R}}=50$ min (Petroleum ether: ethyl acetate 93:7; flow $=3.5 \mathrm{~mL} / \mathrm{min}$ ); $[\alpha]_{\mathrm{D}}^{20}-13^{\circ}$ (c 0.16 , $\mathrm{CHCl}_{3}$ ); IR (film) $v_{\max } 2954,2857,1754,1559,1211,1106 \mathrm{~cm}^{-1}$; ${ }^{1} \mathrm{H}$ NMR $\left(\mathrm{CDCl}_{3}, 500 \mathrm{MHz}\right)$ (see Table 3$) ;{ }^{13} \mathrm{C}$ NMR $\left(\mathrm{CDCl}_{3}\right.$, $125 \mathrm{MHz}$ ) (see Table S3 of the SI); HRESIMS(+): $m / z$ 417.1705 $[\mathrm{M}+\mathrm{Na}]^{+}$(calcd for $\mathrm{C}_{20} \mathrm{H}_{30} \mathrm{O}_{6} \mathrm{NaSi}$, 417.1709); $\mathrm{m} / z 263.0923$ [M+H$\left.\mathrm{C}_{6} \mathrm{H}_{15} \mathrm{SiOH}\right]^{+}$(calcd for $\mathrm{C}_{14} \mathrm{H}_{15} \mathrm{O}_{5}, 263.0919$ ).

\subsubsection{General procedure for the preparation of 6-alkyloxy-4-} (tert-butyldimethylsilyloxy)tetrahydro-2H-pyran-2-ones (3a, 13a-15a). Desymmetrisation of 3-(tert-butyldimethylsilyloxy)pentanedial (1) under solvent-free conditions followed by PCC oxidation

The requisite alcohol (See Table 2 and Scheme 4) $(2.9 \mathrm{mmol})$ was added to a mixture of 3-(tert-butyldimethylsilyloxy)pentanedial (1) ( $1 \mathrm{mmol})$ and $4 \AA$ molecular sieves $(0.5 \mathrm{~g}$ for each $\mathrm{mmol}$ of 1 ) under an argon atmosphere and the mixture stirred for $24 \mathrm{~h}$. The slurry was dissolved in dichloromethane $(20 \mathrm{~mL})$ and added dropwise to a suspension of PCC $(3.5 \mathrm{mmol})$ and powdered molecular sieves $4 \AA$ (twice the weight of the alcohol) in dichloromethane $(70 \mathrm{~mL})$ at room temperature. The reaction was stirred vigorously for $18 \mathrm{~h}$, diethyl ether was then added $(200 \mathrm{~mL})$ and the mixture was stirred for a further $1 \mathrm{~h}$. The suspension was filtered through a pad of silica gel and washed through with a further 
quantity of ether $(200 \mathrm{~mL})$. The ether was removed under reduced pressure to give the crude mixture of tetrahydro- $2 \mathrm{H}$-pyran-2-ones which was purified by column chromatography (petroleum ether $\left./ \mathrm{Et}_{2} \mathrm{O}, 90: 10\right)$, to yield the corresponding tetrahydro- $2 \mathrm{H}$ pyran-2-ones in the ratios and yields shown below and in Table 2 and Scheme 4.

4.3.4.1. ( \pm$)-(4 R(S), 6 S(R))-6$-(benzyloxy)-4-((tert-butyldimethylsilyl)oxy)tetrahydro-2H-pyran-2-one (( \pm$)-13 a) . \quad$ (57.1 mg, 17\%) Colorless oil; IR (film) $v_{\max } 2928,2856,1752,1673,1594$, 1252, $1024 \mathrm{~cm}^{-1} ;{ }^{1} \mathrm{H}$ NMR $\left(\mathrm{CDCl}_{3}, 400 \mathrm{MHz}\right.$ ) (see Table 3$) ;{ }^{13} \mathrm{C}$ NMR $\left(\mathrm{CDCl}_{3}, 100 \mathrm{MHz}\right)$ (see Table S3 of the SI); HRMS $\left(\mathrm{CI}^{+}\right): m / z$ 279.1054 [M-C( $\left.\left.\mathrm{CH}_{3}\right)_{3}\right]^{+}$(calcd for $\mathrm{C}_{14} \mathrm{H}_{19} \mathrm{O}_{4} \mathrm{Si}, 279.1053$ ).

4.3.4.2. ( \pm$)-(4 R(S), 6 S(R))-4-(($ tert-butyldimethylsilyl)oxy)-6-((2methylbenzyl)oxy)tetrahydro-2H-pyran-2-one (( \pm$)-14 a)$. (52.5 mg, 15\%) Colorless oil; IR (film) $v_{\max } 2931,2855,1752$, 1673, 1594, 1249, $1011 \mathrm{~cm}^{-1}$; ${ }^{1} \mathrm{H}$ NMR $\left(\mathrm{CDCl}_{3}, 400 \mathrm{MHz}\right.$ ) (see Table 3); ${ }^{13} \mathrm{C}$ NMR $\left(\mathrm{CDCl}_{3}, 100 \mathrm{MHz}\right)$ (see Table $\mathrm{S} 3$ of the $\mathrm{SI}$ ); HRMS $\left(\mathrm{Cl}^{+}\right): m / z 293.1214\left[\mathrm{M}-\mathrm{C}\left(\mathrm{CH}_{3}\right)_{3}\right]^{+}$(calcd for $\mathrm{C}_{15} \mathrm{H}_{21} \mathrm{O}_{4} \mathrm{Si}$, 293.1209).

4.3.4.3. ( \pm$)-(4 R(S), 6 S(R))-4-(($ tert-butyldimethylsilyl)oxy)-6-((4fluorobenzyl)oxy)tetrahydro-2H-pyran-2-one (( \pm$)-15 a)$. (56.6 mg, 16\%) Colorless oil; IR (film) $v_{\max } 2928,2854,1751$, 1673, 1595, 1223, $1011 \mathrm{~cm}^{-1} ;{ }^{1} \mathrm{H}$ NMR $\left(\mathrm{CDCl}_{3}, 400 \mathrm{MHz}\right)$ (see Table 3); ${ }^{13} \mathrm{C}$ NMR $\left(\mathrm{CDCl}_{3}, 100 \mathrm{MHz}\right)$ (see Table $\mathrm{S} 3$ of the $\mathrm{SI}$ ); HRMS (ESI-QTOF): $\mathrm{m} / \mathrm{z} \quad 377.1546 \quad[\mathrm{M}+\mathrm{Na}]^{+}$(calcd for $\left.\mathrm{C}_{18} \mathrm{H}_{27} \mathrm{O}_{4} \mathrm{FNaSi}, 377.1560\right)$.

4.3.5. General procedure for the preparation of 5-arylalkyloxy3-(hydroxy)pentane-5-lactones (16a-19a). Deprotection with TBAF/ACOH ${ }^{41}$

1.0 M tetrabutylammonium fluoride (TBAF) $(6 \mathrm{mmol})$ in THF was added dropwise to a solution of the corresponding lactones 3a and $( \pm)-13 a-15 a(1 \mathrm{mmol})$ and acetic acid $(5 \mathrm{mmol})$ in dry THF $(6 \mathrm{~mL})$ under an argon atmosphere at room temperature. The reaction was stirred for $16 \mathrm{~h}$ and brine $(10 \mathrm{~mL})$ was then added. The aqueous phase was extracted with ethyl acetate $(3 \times 20 \mathrm{~mL})$, dried over anhydrous sodium sulfate and filtered. Evaporation of the solvent gave a crude product that was purified by silica gel column chromatography (petroleum ether/EtOAc, $80: 20$ ), to afford the corresponding deprotected tetrahydro- $2 \mathrm{H}$ pyran-2-ones ( \pm )-16a-18a and 19a.

4.3.5.1. ( $( \pm)-(4 R(S), 6 S(R))-6$-(benzyloxy)-4-hydroxytetrahydro2H-pyran-2-one (( \pm )-16a). (135.5 mg, 61\%) Colorless oil; IR (film) $v_{\max } 3417,2940,2850,1732,1681,1238,1020 \mathrm{~cm}^{-1} ;{ }^{1} \mathrm{H}$ NMR $\left(\mathrm{CDCl}_{3}, 400 \mathrm{MHz}\right)$ (see Table 4$) ;{ }^{13} \mathrm{C} \mathrm{NMR}\left(\mathrm{CDCl}_{3}, 100 \mathrm{MHz}\right)$ (see Table S4 of the SI); HRMS $\left(\mathrm{Cl}^{+}\right): \mathrm{m} / z 223.0967[\mathrm{M}+\mathrm{H}]^{+}$(calcd for $\mathrm{C}_{12} \mathrm{H}_{15} \mathrm{O}_{4} 223.0970$ ).

4.3.5.2. ( \pm$)-(4 R(S), 6 S(R))-4-h y d r o x y-6-((2-m e t h y l b e n z y l) o x y) t e-$ trahydro-2H-pyran-2-one (( $)$-17a). (134.6 mg, 57\%) Colorless oil; IR (film) $v_{\max } 3398,2945,2879,1727,1570,1224$, $1019 \mathrm{~cm}^{-1}$; ${ }^{1} \mathrm{H}$ NMR $\left(\mathrm{CDCl}_{3}, 400 \mathrm{MHz}\right)$ (see Table 4$) ;{ }^{13} \mathrm{C}$ NMR $\left(\mathrm{CDCl}_{3}, 100 \mathrm{MHz}\right.$ ) (see Table S4 of the SI); HRMS $\left(\mathrm{CI}^{+}\right): \mathrm{m} / z$ $237.1133[\mathrm{M}+\mathrm{H}]^{+}$(calcd for $\mathrm{C}_{13} \mathrm{H}_{17} \mathrm{O}_{4}, 237.1127$ ).

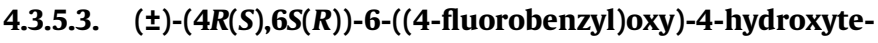

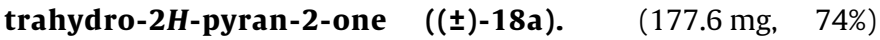
Colouless oil; 74\% yield; IR (film) $v_{\max } 3417,2938,2854,1732$, 1604, 1512, 1223, $1023 \mathrm{~cm}^{-1}$; ${ }^{1} \mathrm{H}$ NMR $\left(\mathrm{CDCl}_{3}, 400 \mathrm{MHz}\right)$ (see Table 4$) ;{ }^{13} \mathrm{C}$ NMR $\left(\mathrm{CDCl}_{3}, 100 \mathrm{MHz}\right)$ (see Table S4 of the $\mathrm{SI}$ ); $\operatorname{HRMS}\left(\mathrm{CI}^{+}\right): \mathrm{m} / z 240.0805[\mathrm{M}]^{+}\left(\right.$calcd for $\left.\mathrm{C}_{12} \mathrm{H}_{13} \mathrm{FO}_{4}, 240.0798\right)$.
4.3.5.4. (4R,6S)-4-hydroxy-6-((R)-1-phenylethoxy)tetrahydro2H-pyran-2-one (19a). (170 mg, 72\%) Colorless oil; HPLC $t_{\mathrm{R}}=38 \mathrm{~min}$ (Petroleum ether: ethyl acetate 60:40; flow $=3.5$ $\mathrm{mL} / \mathrm{min}$ ); $[\alpha]_{\mathrm{D}}^{20}+215.0$ (c 0.5, $\mathrm{CHCl}_{3}$ ); IR (film) $v_{\max } 3398,2927$, 2857, 1752,1560, 1229, $1024 \mathrm{~cm}^{-1} ;{ }^{1} \mathrm{H} \mathrm{NMR}\left(\mathrm{CDCl}_{3}, 400 \mathrm{MHz}\right)$ (see Table 4$) ;{ }^{13} \mathrm{C}$ NMR $\left(\mathrm{CDCl}_{3}, 100 \mathrm{MHz}\right.$ ) (see Table S4 of the SI); $\operatorname{HRMS}\left(\mathrm{CI}^{+}\right): \mathrm{m} / z 221.0818\left[\mathrm{M}-\mathrm{CH}_{3}\right]^{+}$(calcd for $\mathrm{C}_{12} \mathrm{H}_{13} \mathrm{O}_{4}, 221.0814$ ).

4.3.6. Preparation of $(4 S, 6 S)$-4-hydroxy-6-( $(R)-1-$ phenylethoxy)tetrahydro-2H-pyran-2-one $(19 b)^{41}$

1.0 M tetrabutylammonium fluoride (TBAF) $(0.6 \mathrm{mmol})$ in THF was added dropwise to a solution of the corresponding tetrahydro-2H-pyran-2-one $\mathbf{3 b}(0.05 \mathrm{mmol})$ and acetic acid $(0.5 \mathrm{mmol})$ in dry THF $(0.6 \mathrm{~mL})$ under an argon atmosphere at room temperature. The reaction was stirred for $16 \mathrm{~h}$ and brine $(10 \mathrm{~mL})$ was then added. The aqueous phase was extracted with ethyl acetate $(3 \times 20 \mathrm{~mL})$, dried over anhydrous sodium sulfate and filtered. Evaporation of the solvent gave a crude product that was purified by silica gel column chromatography (petroleum ether/EtOAc, $80: 20$ ), to afford the corresponding deprotected tetrahydro- $2 \mathrm{H}$ pyran-2-one 19b (8.5 mg, 72\%) as a colorless oil; HPLC $t_{\mathrm{R}}=47 \mathrm{~min}$ (Petroleum ether: ethyl acetate 60:40; flow $=3.5 \mathrm{~mL} / \mathrm{min}$ ); $[\alpha]_{\mathrm{D}}^{20}+130.0\left(c \quad 0.1, \mathrm{CHCl}_{3}\right.$ ); IR (film) $v_{\max }$ 3412, 2927, 2857, 1752, 1560, 1229, $1024 \mathrm{~cm}^{-1}$; ${ }^{1} \mathrm{H}$ NMR $\left(\mathrm{CDCl}_{3}\right.$ $400 \mathrm{MHz}$ ) (see Table 4); ${ }^{13} \mathrm{C}$ NMR $\left(\mathrm{CDCl}_{3}, 100 \mathrm{MHz}\right.$ ) (see Table S4 of the SI); HRMS $\left(\mathrm{CI}^{+}\right): \mathrm{m} / \mathrm{z} 221.0817\left[\mathrm{M}-\mathrm{CH}_{3}\right]^{+}$(calcd for $\left.\mathrm{C}_{12} \mathrm{H}_{13} \mathrm{O}_{4}, 221.0814\right)$.

\section{Acknowledgments}

This research was supported by grants from Junta de Andalucía (P07-FQM-02925) and, in part, from MINECO (AGL2012-39798C02-01) (Spain). A.J.M.S. gratefully acknowledges a RSC research fund grant (UK). J.M.B. thanks Junta de Andalucía for Research Fellowship. Use of NMR and mass spectrometry (QTOF) facilities at Servicios Centrales de Investigación Científica y Tecnológica (SC-ICYT) of the University of Cádiz is acknowledged.

\section{Supplementary data}

Supplementary data (percentage of inhibition, $B$. cinerea and $C$. gloeosporioides) for compounds $\mathbf{1 6 a - 1 9 a},{ }^{13} \mathrm{C}$ spectroscopic data for 3a and b, 5a, 5c, 13a-19a and 19b together with ${ }^{1} \mathrm{H}$ and ${ }^{13} \mathrm{C}$ NMR spectra for compounds $3 \mathbf{a}$ and $\mathbf{b}, 5 \mathbf{5}, 5 \mathbf{c}, 13 \mathbf{a}-19 \mathbf{a}$ and 19b.) associated with this article can be found, in the online version, at http://dx.doi.org/10.1016/j.bmc.2015.04.048.

\section{References and notes}

1. Droby, S.; Lichter, A. In Botrytis: biology, pathology and control; Elad, Y. Williamson, B., Tudzynski, P., Delen, N., Eds.; Springer: Dordrecht, Netherlands, 2007; pp 349-367.

2. Williamson, B.; Tudzynski, B.; Tudzynski, P.; Van Kan, J. A. L. Mol. Plant Pathol. 2007, 8, 561 .

3. Leroux, P. Botrytis: Biology, Pathology and Control; Botrytis: Biology; Springer: Netherlands, 2007. pp 195-222.

4. Smith, C. M. Fungicide Resistance in North America In Delp, C. J., Ed.; American Phytopathological Society Press: St. Paul, Minnesota, USA, 1988; pp 23-24.

5. Brent, K. J.: Hollomon, D. W. Fungicide Resistance in Crop Pathogens: How Can It Be Managed?, 2nd Rev ed. Online; Resistance Action Committee (FRAC). CropLife International: Brussels, Belgium, 2007. pp. 36-37.

6. Spadaro, D.; Gullino, M. L. Int. J. Food Microbiol. 2004, 91, 185.

7. Ragsdale, N. N. Annu. Rev. Phytopathol. 2000, 38, 577.

8. Rosslenbroich, H.; Stuebler, D. Crop Prot. 2000, 19, 557.

9. Terry, L. A.; Joyce, D. C. Pest Manage. Sci. 2000, 56, 989.

10. van Kan, J. A. L. Trends Plant Sci. 2006, 11, 247.

11. Sharon, A.; Elad, Y.; Barakat, R.; Tudzynski, P. In Botrytis: Biology, Pathology and Control; Elad, Y., Williamson, B., Tudzynski, P., Delen, N., Eds.; Springer: Netherlands, Dordrecht, The Netherlands, 2007; pp 163-179.

12. Choquer, M.; Fournier, E.; Kunz, C.; Levis, C.; Pradier, J.; Simon, A.; Viaud, M. FEMS Microbiol. Lett. 2007, 277, 1 
13. Collado, I. G.; Macias-Sanchez, A. J.; Hanson, J. R. Nat. Prod. Rep. 2007, 24, 674. references cited therein.

14. Bradshaw, A. P. W.; Hanson, J. R.; Nyfeler, R.; Sadler, I. H. J. Chem. Soc., Perkin Trans.1 1982, 2187.

15. Siewers, V.: Viaud, M.: Jimenez-Teja, D.: Collado, I. G.: Schulze Gronover, C.; Pradier, J. M.; Tudzynsk, B.; Tudzynski, P. Mol. Plant-Microbe Interact. 2005, 18 , 602.

16. Pinedo, C.; Wang, C. M.; Pradier, J. M.; Dalmais, B.; Choquer, M.; Le Pêcheur, P.; Morgant, G.; Collado, I. G.; Cane, D. E.; Viaud, M. L. ACS Chem. Biol. 2008, 3, 791.

17. Wang, C. M.; Hopson, R.; Lin, X.; Cane, D. E. J. Am. Chem. Soc. 2009, 131, 8360.

18. Shiina, I.; Fukui, H. Chem. Commun. 2009, 385.

19. Reino, J. L.; Durán-Patrón, R. M.; Daoubi, M.; Collado, I. G.; Hernández-Galán, R. J. Org. Chem. 2006, 71, 562.

20. Massaroli, M.; Moraga, J.; Bastos Borges, K.; Ramírez-Fernández, J.; Viaud, M.; González Collado, I.; Durán-Patrón, R.; Hernández-Galán, R. ChemBioChem 2013, 14, 132.

21. Dalmais, B.; Schumacher, J.; Moraga, J.; Le Pêcheur, P.; Tudzynski, B.; Collado, I. G.; Viaud, M. Mol. Plant Pathol. 2011, 12, 564.

22. Heller, J.; Ruhnke, N.; Espino, J. J.; Massaroli, M.; Collado, I. G.; Tudzynski, P. Mol. Plant Microbe Interact. 2012, 25, 802.

23. Amselem, J.; Cuomo, C. A.; van Kan, J. A. L.; Viaud, M.; Benito, E. P.; Couloux, A.; Coutinho, P. M.; de Vries, R. P.; Dyer, P. S.; Fillinger, S.; Fournier, E.; Gout, L.; Hahn, M.; Kohn, L.; Lapalu, N.; Plummer, K. M.; Pradier, J. M.; Quévillon, E; Sharon, A.; Simon, A.; Have, A.; Tudzynski, B.; Tudzynski, P.; Wincker, P.; Andrew, M.; Anthouard, V.; Beever, R. E.; Beffa, R.; Benoit, I.; Bouzid, O.; Brault B.; Chen, Z.; Choquer, M.; Collémare, J.; Cotton, P.; Danchin, E. G.; Da Silva, C.; Gautier, A.; Giraud, C.; Giraud, T.; Gonzalez, C.; Grossetete, S.; Güldener, U.; Henrissat, B.; Howlett, B. J.; Kodira, C.; Kretschmer, M.; Lappartient, A.; Leroch, M.; Levis, C.; Mauceli, E.; Neuvéglise, C.; Oeser, B.; Pearson, M.; Poulain, J.; Poussereau, N.; Quesneville, H.; Rascle, C.; Schumacher, J.; Ségurens, B.; Sexton, A.; Silva, E.; Sirven, C.; Soanes, D. M.; Talbot, N. J.; Templeton, M.; Yandava, C.; Yarden, O.; Zeng, Q.; Rollins, J. A.; Lebrun, M. H.; Dickman, M. PLoS Genet. 2011 7, e1002230.

24. Ascari, J.; Boaventura, M. A. D.; Takahashi, J. A.; Duran-Patron, R.; HernandezGalan, R.; Macias-Sanchez, A. J.; Collado, I. G. J. Nat. Prod. 2013, 1016, 76 references cited therein.

25. Endo, A. Nat. Med. (N.Y., NY, U.S.) 2008, 1050, 14

26. Endo, A. Proc. Jpn. Acad. 2010, 86(Ser.B), 484.

27. Salakhutdinov, N. F.; Laev, S. S. Bioorg. Med. Chem. 2014, 22, 3551.

28. Jain, K. S.; Kathiravan, M. K.; Somani, R. S.; Shishoo, C. J. Bioorg. Med. Chem. 2007, 15, 4674.
29. Macreadie, I. G.; Johnson, G.; Schlosser, T.; Macreadie, P. I. FEMS Microbiol. Lett. 2006, 262, 9

30. Cazar, M. E.; Schmeda-Hirschmann, G.; Astudillo, L. World J. Microbiol. Biotechnol. 2005, 21, 1067.

31. Wess, G.; Granzer, E.; Beck, G.: Lau, H. H. Chem. Abstr. 1987, 106, 196256.

32. Willis, M. C. J. Chem. Soc., Perkin Trans.1 1999, 1765.

33. Dodd, K.; Morton, D.; Worden, S.; Narquizian, R.; Nelson, A. Chem. Eur. J. 2007, 13, 5857.

34. Buckley, S. L. J.; Drew, M. G. B.; Harwood, L. M.; Macías-Sánchez, A. J. Tetrahedron Lett. 2002, 43, 3593.

35. Brandänge, S.; Färnbäck, M.; Leijonmarck, H.; Sundin, A. J. Am. Chem. Soc. 2003, $125,11942$.

36. See other preparative procedures via protected glutarate ester reduction: (a) Mitton-Fry, M. J.; Cullen, A. J.; Sammakia, T. Angew. Chem., Int. Ed. 2007, 46, 1066; (b) Zhang, Y.; Arpin, C. C.; Cullen, A. J.; Mitton-Fry, M. J.; Sammakia, T. J. Org. Chem. 2003, 7, 1027; A related p-methoxybezoate protected dialdehyde has been previously obtained through a similar methodology: Shepherd, J. N.; Myles, D. C. Org. Lett 2011, 76, 7641.

37. Catalysts for desymmetrisation of meso and prochiral anhydrides Cheng, Y.; McDaid, P.; Deng, L. Chem. Rev. 2003, 103, 2965.

38. Catalyst for desymmetrisation of meso epoxides Bartoli, G.; Bosco, M.; Carlone, A.; Locatelli, M.; Massaccesi, M.; Melchiorre, P.; Sambri, L. Org. Lett. 2004, 6, 2173.

39. Smith, B. M.; Kubczyk, T. M.; Graham, A. E. Tetrahedron 2012, 68, 7775.

40. Although yields of lactones $\mathbf{3 a}$ and $\mathbf{3 b}$ were not significantly improved, an advantageous aspect of the reactions in solvent-free conditions is that they can be carried out at room temperature while, for reaction to proceed in dry THF, it was necessary to heat the reaction to reflux.

41. Smith, A. B.; Ott, G. R. J. Am. Chem. Soc. 1996, 118, 13095.

42. Patil, I. S.; Kulkarni, S.; Hedge, R. K. Pesticides 1986, 30.

43. Lorito, M.; Woo, S. L.; Harman, G. E.; Monte, E. Annu. Rev. Phytopathol. 2010, 48, 395.

44. Lorito, M.; Peterbauer, C.; Hayes, C. K.; Harman, G. E. Microbiology 1994, 140, 623.

45. Lorito, M.; Woo, S. L.; D’Ambrosio, M.; Harman, G. E.; Hayes, C. K.; Kubicek, C. P.; Scala, F. Mol. Plant-Microbe Interact. 1996, 9, 206.

46. García-Pajón, C. M.; Collado, I. G. Nat. Prod. Rep. 2003, 20, 426.

47. Phillips, J. N.; Vincent, J. M. Nature 1948, 161, 210.

48. Austin, K. A. B.; Banwell, M. G.; Willis, A. C. Org. Lett. 2008, 10, 4465. 